\title{
CLUSTERING IN WIRELESS SENSOR NETWORK: A REVIEW
}

\author{
Harpinderjeet Kaur Sekhon (1), Vikramjit Singh ${ }^{(2)}$ \\ (1) Research Scholar, Department of Computer Science and Engineering, NWIET, Moga \\ (2) Assistant Professor, Department of Computer Science and Engineering, NWIET, Moga
}

\begin{abstract}
WSNs are evolving as both a vital new domain in the IT environment and a hot research including system design, networking, and distributed algorithms, programming models, data management, security and social components. Wireless sensor networks are rapidly picking up the popularity as they are potentially low cost solutions. The fundamental thought of sensor network is to scatter minor sensing gadgets over a particular geographic zone for some specific purposes like target tracking, surveillance, environmental screening and so on. These tiny devices are equipped for sensing a few progressions of parameters and communicating with different units. Since the network is wireless, the nodes communicate with each other via means of wireless. The wireless sensor network is interfaced with the outside world by a gateway server or controlling server. This paper focuses on the detailed survey of various clustering approaches used in wsn.
\end{abstract}

\section{Keywords}

Wireless sensor network, wsn, cluster head, base station, sensor node

\section{INTRODUCTION}

A Wireless Sensor Network or WSN is supposed to be made up of a large number of sensors and at least one base station The sensors are autonomous small devices with several constraints like the battery power, computation capacity, communication range and memory. They also are supplied with transceivers to gather information from its environment and pass it on up to a certain base station, where the measured parameters can be stored and available for the end user. In most cases, the sensors forming these networks are deployed randomly and left unattended to and are expected to perform their mission properly and efficiently [1]. As a result of this random deployment, the WSN has usually varying degrees of node density along its area. Sensor networks are also energy constrained since the individual sensors, which the network is formed with, are extremely energy-constrained as well. The communication devices on these sensors are small and have limited power and range. Both the probably difference of node density among some regions of the network and the energy constraint of the sensor nodes cause nodes slowly die making the network less dense. Also, it is quite common to deploy WSNs in harsh environment, what makes many sensors in operable or faulty. For that reason, these networks need to be fault-tolerant so that the need for maintenance is minimized [2]. Typically, the network topology is continuously and dynamically changing, and it is actually not a desired solution to replenish it by infusing new sensors instead the depleted ones. A real and appropriate solution for this problem is to implement routing protocols that perform efficiently and utilizing the less amount of energy as possible for the communication among nodes. A wireless sensor network is a technology that emerges as a consequence of the evolution of network technology along with microelectronics and micromechanical devices. It is a new concept, a view towards the future, a clear consequence of the new steps forward in the communications field. In few words a wireless sensor network, is a network that could contain from a couple to many small nodes with sensors attached and communications capabilities to transmit and receive information [3.

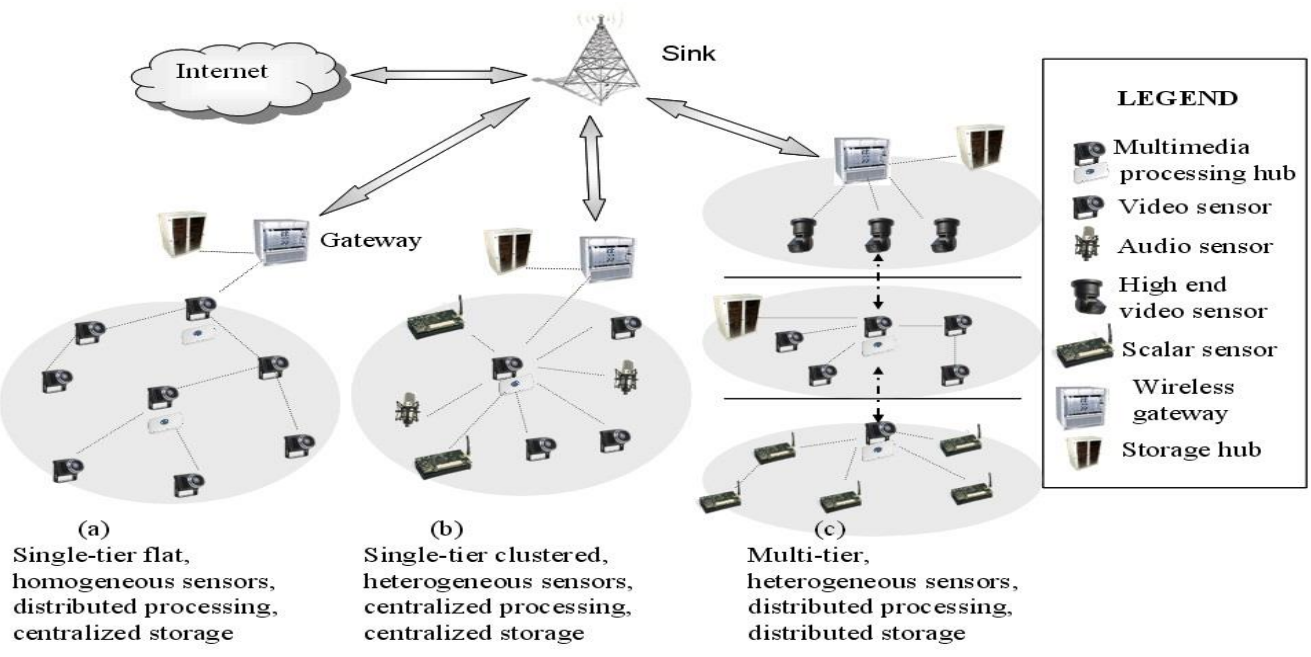

Figure 1. Wireless Sensor Network

With the help of this technology such systems will now be capable of raising a huge amount of data and results which will be available for analysis in real time. This could lead to a new era of monitoring and controlling processes as they are occurring, something that it was not possible before in some cases without the intervention of persons and complex equipment. Another advantage in the use of wireless technology is the reduction in cost that cabling deployment has in current systems and also the possibility of performing measurements in inaccessible places. In ad-hoc network each and 
every node can communicate with each other without any fixed infrastructure [4]. This is actually one of the features that differentiate between ad-hoc and other wireless technology like cellular networks and wireless LAN which actually required infrastructure based communication like through some base station. Wireless sensor network is one of the category belongs to ad-hoc networks. Sensor network contains multiple nodes. Here actually the node has a specific name that is "Sensor" because these nodes are equipped with smart sensors. A sensor node is a device that converts a sensed characteristic like temperature, vibrations, pressure into a form recognize by the users. Wireless sensor networks nodes are less mobile than ad-hoc networks. So, mobility in case of ad-hoc is more. In wireless sensor network data are requested depending upon certain physical quantity. So wireless sensor network is data centric [5]. A sensor consists of a transducer, an embedded processor, small memory unit and a wireless transceiver and all these devices run on the power supplied by an attached. The main entities that constitute a sensor network are:

i. Sensor nodes are the main component of sensor network. Their main objectives are making discrete, local measurement about phenomenon surrounding these sensors, forming a wireless network by communicating over a wireless medium, and collect date and rout data back to the user via sink (Base Station).

ii. The sink (Base Station) communicates with the user via internet or satellite communication. It is located near the sensor field or well-equipped nodes of the sensor network. Collected data from the sensor field routed back to the sink by a multi-hop infrastructure less architecture through the sink.

iii. Phenomenon which is an entity of interest to the user to collect measurements about. This phenomenon sensed and analyzed by the sensor nodes.

iv. The user who is interested in obtaining information about specific phenomenon to measure/monitor its behavior.

\section{SENSOR NODES}

Sensors nodes are typically built of few sensors and a mote unit as shown in figure 1.2. The sensor nodes are scattered in a special domain called sensor network. These nodes mainly have the capability to collect data, analyze them and route them to a sink point i.e. a sensor is a device which senses the information and pass it on to mote. Sensors are typically used to measure the changes in physical environmental parameters like temperature, pressure, humidity, sound, vibration and changes in the health parameter of person e.g. blood pressure and heartbeat. MEMS based sensor have found good use in sensor nodes. A mote consists of processor, memory, battery, A/D converter for connecting to a sensor and a radio transceiver for forming an ad hoc network. A mote and sensor together form a Sensor Node. A sensor network is a wireless ad-hoc network of sensor nodes. Each sensor node can support a multi-hop routing algorithm and function as forwarder for relaying data packets to a base station.

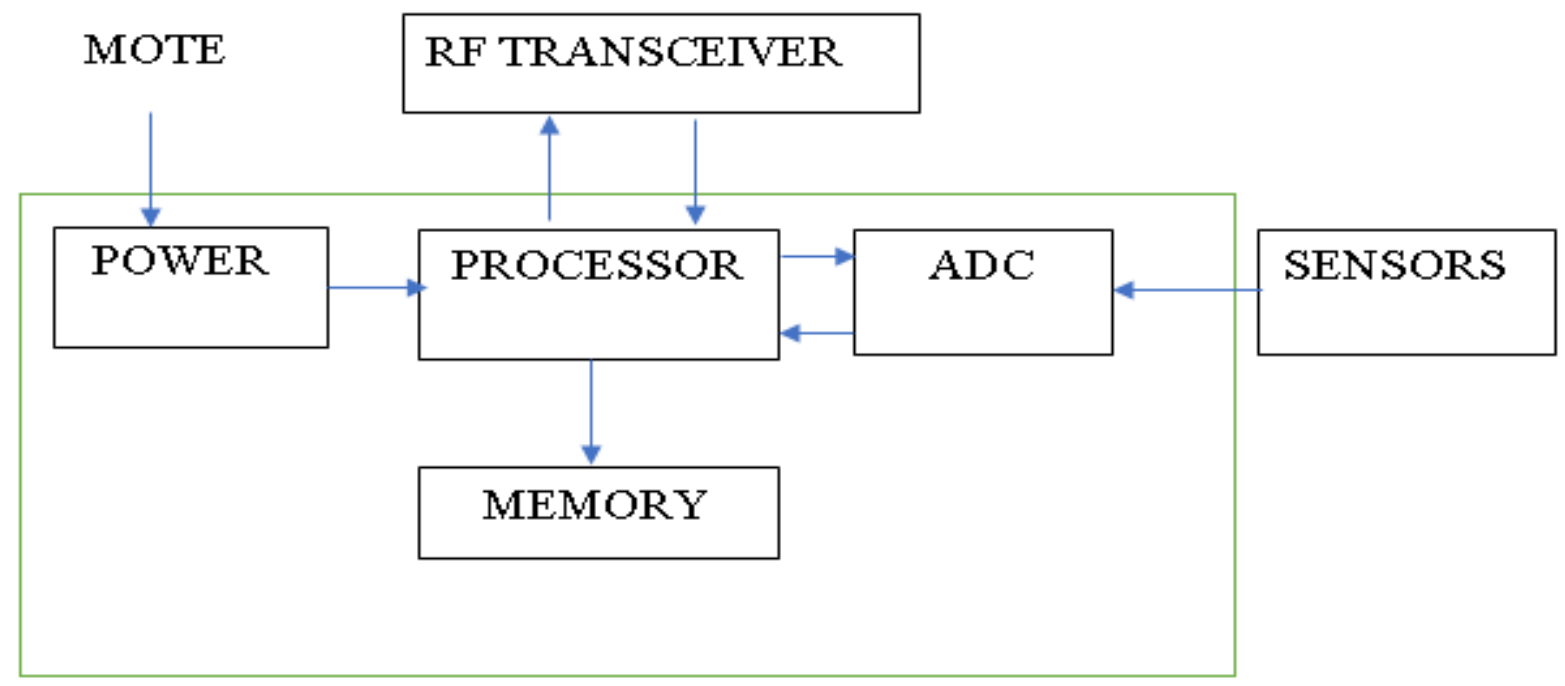

Figure 2. Block Diagram of Sensor Node

The major characteristics of the sensor node used to evaluate the performance of WSN are [6]:

i. Fault tolerance: Each node in the network is prone to unanticipated failure. Fault tolerance is the capability to maintain sensor network functionalities without any break due to sensor node failures.

ii. Mobility of nodes: In order to increase the communication efficiency, the nodes can move anywhere within the sensor field based on the type of applications.

iii. Communication failures: If any node in the WSN fails to exchange data with other nodes, it should be informed without delay to the base station or gateway node.

iv. Scalability: The number of sensor nodes in a sensor network can be in the order of hundreds or even thousands. Hence, WSN designed for sensor networks is supposed to be highly scalable.

v. Independency: The WSN should have the capability to work without any central control point.

vi. Utilization of sensors: The sensors should be utilized in a way that produces the maximum performance with less energy. 


\section{BASE STATION}

It is an extraordinary type of node having high computational energy and processing capability. A base station links the sensor network to another network. It consists of a processor, radio board, antenna and USB interface board. It is preprogrammed with low-power mesh networking software for communication with wireless sensor nodes. Deployment of the base station in a wireless sensor network is very important as all the sensor nodes handover their data to the base station for processing and decision making [7]. Energy conservation, coverage of sensor nodes and reliability issues are taken care of during deployment of base station in sensor network. Generally base stations are assumed static in nature but in some scenarios, they are assumed to be mobile to collect the data from sensor nodes.

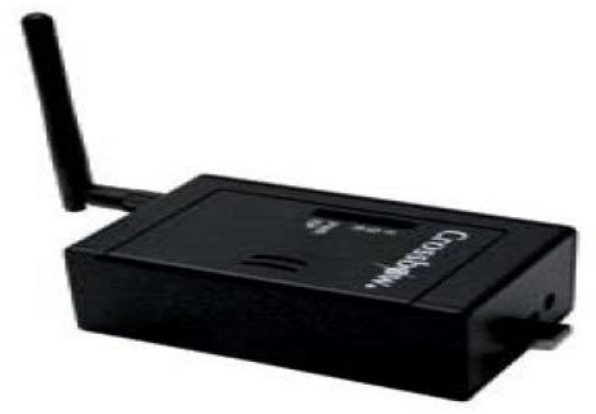

Figure 3. Base Station

WSNs are evolving as both a vital new domain in the IT environment and a hot research including system design, networking, and distributed algorithms, programming models, data management, security and social components. Wireless sensor networks are rapidly picking up the popularity as they are potentially low cost solutions. The fundamental thought of sensor network is to scatter minor sensing gadgets over a particular geographic zone for some specific purposes like target tracking, surveillance, environmental screening and so on. These tiny devices are equipped for sensing a few progressions of parameters and communicating with different units. Since the network is wireless, the nodes communicate with each other via means of wireless. The wireless sensor network is interfaced with the outside world by a gateway server or controlling server. A proper and efficient access method is needed to be established for the gateway server to access the nodes in the network. In wireless sensor network, all nodes work in a very cooperative manner to carry out their tasks and the gateway server acts as an interface between the nodes and the server for information access. Like any network, the wireless nodes need to have unique ID for efficient and proper communication. When the ID of a node is broadcast, the respective node needs must be capable to recognize the transmission as destined to it for establishing communication for follow-up transmission of data and at the same time, the other nodes must be able to figure out that the broadcast ID is not destined to them and must be capable of ignoring the request [9]. In this way, a handshaking takes place between the gateway server and the node of interest, before any communication takes place. Wireless sensor network has two types:

i. Structured

ii. Unstructured

Unstructured network is a collection of sensor nodes deployed in adhoc manner into a region. Once deployed, the network is absent unattended perform monitoring and reporting functions. While in a structured wireless sensor network, the sensor nodes are deployed in predesigned manner [10]. The structured wireless sensor network is more advantageous as some nodes can be deployed with lower network maintenance and management cost.

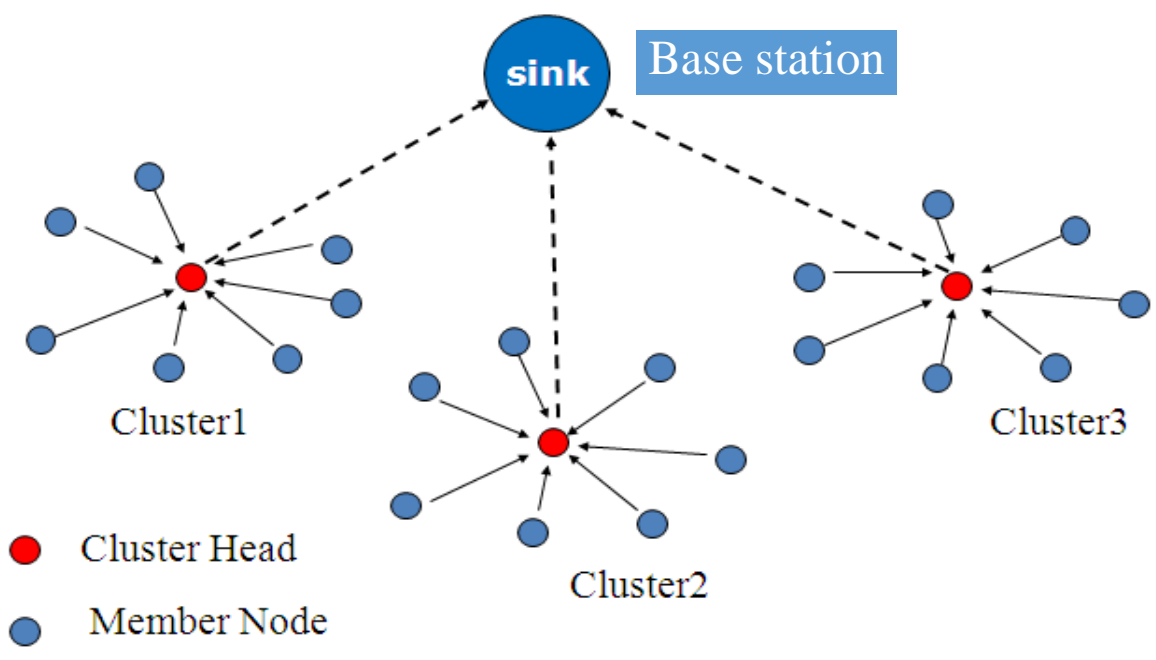

Figure 4. General Architecture of Sensor Network 


\section{CHARACTERISTICS OF WIRELESS SENSOR NETWORKS}

i. Low cost: In the WSN normally hundreds or thousands of sensor nodes are deployed to measure any physical environment. In order to reduce the overall cost of the whole network the cost of the sensor node must be kept as low as possible.

ii. Energy efficient: Energy in WSN is used for different purpose such as computation, communication and storage. Sensor node consumes more energy compare to any other for communication. If they run out of the power they often become invalid as we do not have any option to recharge [12]. So, the protocols and algorithm development should consider the power consumption in the design phase.

iii. Computational power: The node has normally limited computational capabilities as the cost and energy need to be considered.

iv. Communication Capabilities: WSN typically communicate using radio waves over a wireless channel. It has the property of communicating in short range. The communication channel can be either bidirectional or unidirectional. With the unattended and hostile operational environment, it is difficult to run WSN smoothly. So, the hardware and software for communication must have to consider the robustness, security and resiliency.

v. Distributed sensing and processing: The large number of sensor node is distributed uniformly or randomly. WSNs each node is capable of collecting, sorting, processing, aggregating and sending the data to the sink. Therefore the distributed sensing provides the robustness of the system.

vi. Dynamic network topology: In general WSN are dynamic network. The sensor node can fail for battery exhaustion or other circumstances, communication channel can be disrupted as well as the additional sensor node may be added to the network that result the frequent changes in the network topology. Thus, the WSN nodes have to be embedded with the function of reconfiguration, self-adjustment.

vii. Multi-hop communication: A large number of sensor nodes are deployed in WSN. So, the feasible way to communicate with the sinker or base station is to take the help of an intermediate node through routing path. If one need to communicate with the other node or base station which is beyond its radio frequency it must communicate through the multi-hop route by intermediate node.

viii. Application oriented: WSN is different from the conventional network due to its nature. It is highly dependent on the application ranges from military, environmental as well as health sector. The nodes are deployed randomly and spanned depending on the type of use.

ix. Small physical size: Sensor nodes are generally small in size with the restricted range. Due to its size its energy is limited which makes the communication capability low.

\section{APPLICATIONS OF WIRELESS SENSOR NETWORKS}

Sensor networks are applied in a wide range of areas, such as military applications, public safety, medical, surveillances, environmental monitoring, commercial applications, habitat and tracking. In general, sensor networks are ubiquitous since they support opportunities for the interaction between humans and their physical world [13]. In addition, sensor networks are expected to contribute significantly to pervasive computing and space exploration. Moreover, other applications for wireless sensor networks can be seen in environmental monitoring and control field, high-security smart homes, tracking, and identifications and personalization.

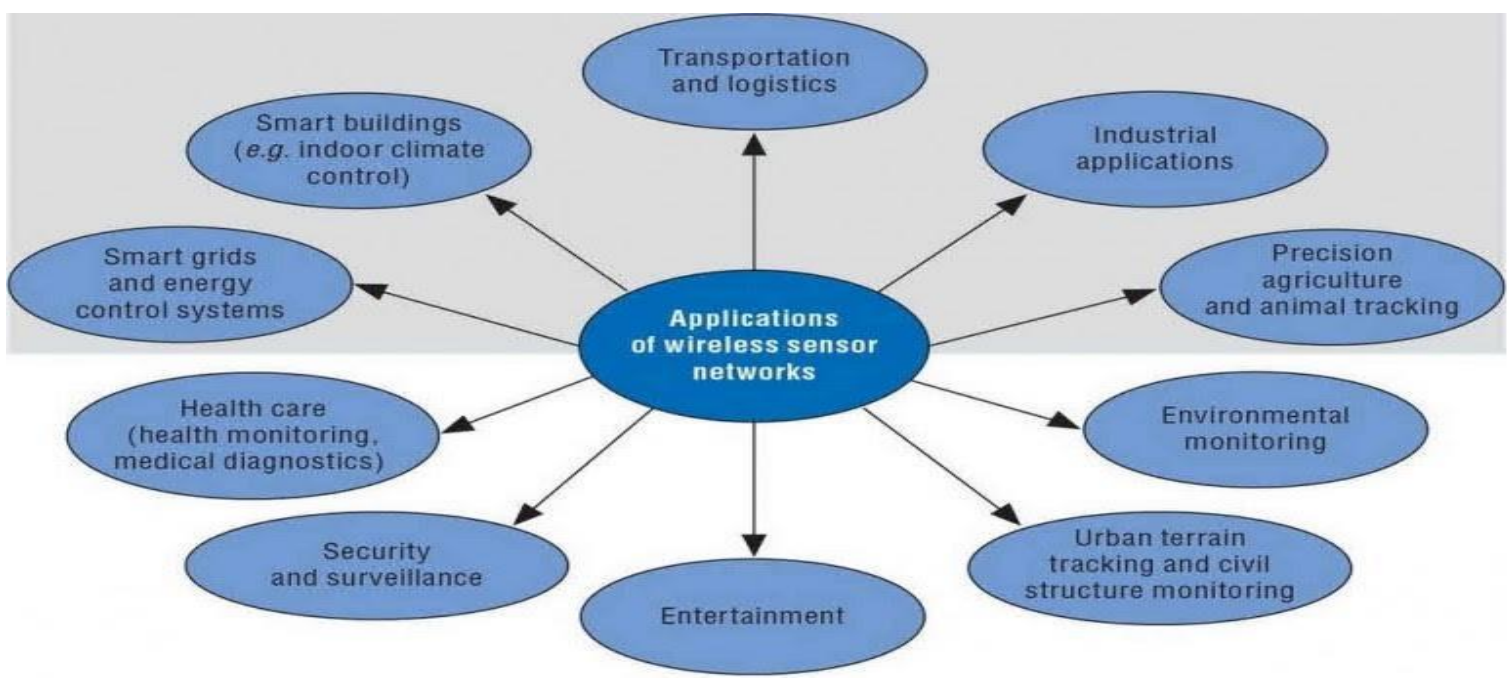

Figure 5. Application Space

i. Military Applications: Wireless sensor networks are becoming an integral part of military command, control, communication, computing, intelligence, surveillance and targeting systems. The rapid deployment, fault tolerance and self-organization characteristics of sensor networks make them a very promising sensing technique for military [4]. 
ii. Environmental Applications: Some environmental applications of sensor network include tracking the movement of birds, small animals and insects; monitoring environmental conditions that affect crops and livestock ; irrigation; macro instruments for large scale earth monitoring and planetary exploration; chemical/biological detection; precision agriculture; Earthen and environmental monitoring in marine, soil and atmospheric contexts ; forest fire detection and meteorological and geophysical research; flood detection; bio complexity mapping of the environment and pollution study.

iii. Health Application: Some of the applications are providing interfaces for the disabled; integrated patient monitoring; diagnostics; drug administration in hospital; monitoring the movements and internal process of insects or others mall animals; Tele-monitoring of human physiological data; and tracking and monitoring doctors and patients inside a hospital. For example, sensors, can be deployed in a patient's home to monitor the behaviors of the patient. It can alert doctors when the patient falls and requires immediate medical attention.

iv. Home Applications: Wireless sensor networks can be used to provide more convenient and intelligent living environments for human beings. Home automation; as technology advances, smart sensor nodes and actuators can be buried appliances, such as vacuum cleaners, microwave ovens, refrigerators and VCRs.

v. Agriculture: By using wireless sensor networks within the agricultural industry is increasingly common; using a wireless network frees the farmer from the maintenance of wiring in a difficult environment [5]. Gravity feed water systems can be monitored using pressure transmitters to monitor water tank levels, pumps can be controlled using wireless $\mathrm{I} / \mathrm{O}$ devices and water use can be measured and wirelessly transmitted back to a central control center for billing. Irrigation automation enables more efficient water use and reduces waste.

vi. Structural Monitoring: Wireless sensors can be used to monitor the movement within buildings and infrastructure such as bridges, flyovers, embankments, tunnels etc. enabling Engineering practices to monitor assets remotely without the need for costly site visits, as well as having the advantage of daily data, whereas traditionally this data was collected weekly or monthly, using physical site visits, involving either road or rail closure in some cases [6]. It is also far more accurate than any visual inspection that would be carried out.

\section{CLUSTERING IN WIRELESS SENSOR NETWORKS}

Despite the innumerable applications of WSNs, these networks have several restrictions, e.g., limit deanery supply, limited computing power, and limited bandwidth of the wireless links connecting sensor nodes. Wireless sensor networks are energy-limited and application-specific. To reduce the cost and size of these sensors, they are equipped with small batteries. Each battery-power sensor node is a constrained device with a relatively small memory resources, restricted computational power, and limited communication capability. A sensor node in such a network can interact directly only with other sensors lying within a small range [7]. To achieve communication within sensors out of each other's communication range, the sensors form a multi-hop communication network. While concentrating on the general network design issues in wireless sensor networks, there are numerous vital aspects that need attention such as the small size of the sensor node, its hardware complexity and ultra-low energy consumption. Among them, energy efficiency is considered as the key design objective, since a sensor node can only be furnished with a limited energy supply. Clustering is one of the design methods used to manage the network energy consumption efficiently, by minimizing the number of nodes that take part in long-distance communication with the base station and distributing the energy consumption evenly among the nodes in the network. In Clustering, the sensor nodes are apportioned into distinctive groups [8]. Each one cluster is overseen by a node alluded as group head $(\mathrm{CH})$ and different nodes are alluded as cluster nodes. Group nodes don't speak straightforwardly with the sink node. They need to pass the gathered information to the cluster head. Group head will total the information, gained from cluster nodes and transmits it to the base station. In this way, it minimizes number of messages imparted to base station. The primary focus of cluster based routing is to effectively maintain the energy usage of sensor nodes by including them in multi-hop communication inside a specific cluster. In Clustering, the sensor nodes are apportioned into distinctive groups.

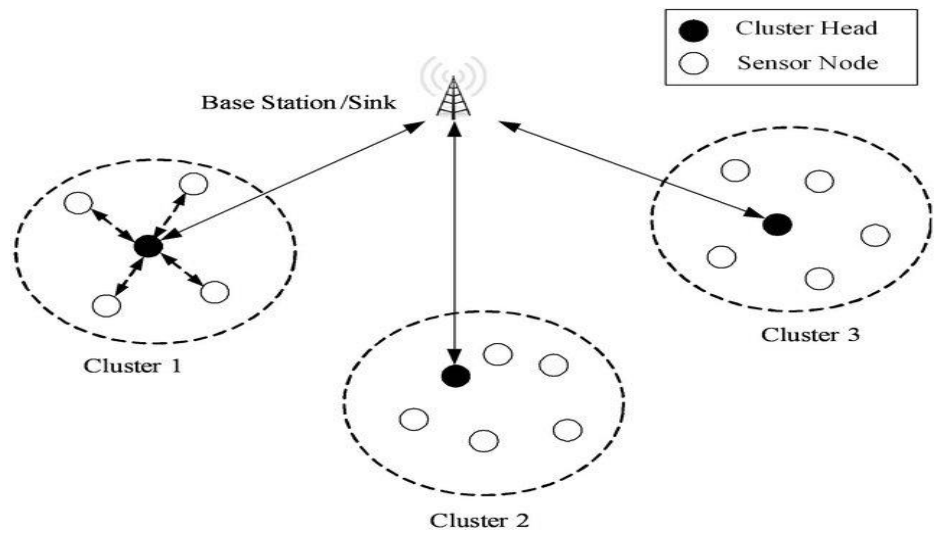

Figure 6. Clustering in WSN

\section{LITERATURE SURVEY}

The needed detailed literature survey, to get preliminary knowledge and search scope of investigation, to implement Low energy adaptive clustering hierarchy, is explained in this chapter. This synopsis presents investigational studies in several energy efficient routing algorithms and its general purpose. Yu Xiuwu et al (2016) proposes a routing protocol that is based 
on monitoring area partition clustering for energy-balanced (EBPC). They divided monitoring area into several virtual partitions, controlled the number of common nodes and clusters by data fusion rate, and forwarded cluster heads data to the next partitions with minimum path selection coefficient. Samaresh Bera et al (2016) proposes a software-defined wireless sensor network architecture (Soft-WSN) -an effort to support application-aware service provisioning in Internet of Things (IOT). Detailed architecture of the proposed system is presented involving the application, control, and infrastructure layers to enable software-defined networking (SDN) in loT. Alduais et al (2016) specifies that WSN has become an enabler technology for the IOT applications which extend the physical reach of the monitoring capability. WSN as it is, possess several constraints such as limited energy availability, low memory size, and low processing speed, which are the principal obstacles to designing efficient management protocols for WSNs, what more if it concerns WSN-IOT integration. Omkar Singh et al (2016) compares three well accepted WSN routing protocols namely LEACH, FAIR and SEP for their energy pattern in heterogeneous scenario. Heterogeneity is introduced in terms of initial random energy values given to the sensor nodes. All simulations are done in MATLAB. Different parameters are used for checking the efficacy of the considered routing protocol for H-WSN. Rani Kumari et al (2016) analyzes the performance of the various routing protocols of the wireless network in the WBAN and WSN. Moreover, the paper also compares their performance in the same network and in different network using different parameters i.e. PDR, latency and throughput etc. The research signifies that the wireless protocols needs updating to perform well in the WBAN. Hector Kaschel et al (2016) describes the mechanisms to achieve energy efficiency in a WSN, focusing particularly on routing protocols. Based on the state of the art, is presented a complete taxonomy of routing protocols used in WSN exposing their level of energy efficiency. Asdianur et al (2016) studied effectiveness in using Compressive Sensing (CS) algorithm in order to reduce measuring in IEEE 802.15.4 Standard Wireless Sensor Network (WSN). As well known, in common WSN work system, Base Station (BS) gather some information from available nodes, which the process itself consumes a lot of energy from each node. Mohd Zaki Shahabuddin et al (2016) proposes fundamental modelling of topology control algorithm to conserve individual WSN node's energy, and at the same time preserving the graph connectivity. The proposed topology control algorithm consists of three phases: 1 . Identifying connecting nodes at maximum transmission, 2. pairing nodes with shortest algorithm/minimum energy level, 3. Calculating/setting minimum power transmission per-node for energy conservation. The algorithm works-out locally and dispenses full graph connectivity, and theoretically would be able to reduce WSN control overhead. Abhay kumar et al (2016) presents a novel scheme to improve the lifetime of a sensor node by optimizing the size of the packet such that there is no loss of data along with providing an increased lifetime for the nodes. Wireless sensor network is a rapid developing area with diverse applications. Smartness and interoperability of network keeps it in high demand and hence comes need for the efficiency of the system. The most important limitation on sensor node is the low power consumption. Sensor nodes carry inadequate, generally irreplaceable power sources. Therefore, while traditional networks aim to achieve high quality of service (QoS), wireless sensor network protocols must emphasis predominantly on power conservation. B. Bengherbia et al (2016) states that the wireless sensor network (WSN) represent fertile area for research and exploration, especially with enormous development in modern electronic systems, where it becomes an alternative to traditional wired systems. The wireless stems are less energy-consumptive and inexpensive, in addition it helps to reduce the complexity of wiring. Wireless sensor networks require the design of high density computing, an energy-efficient and a flexible node architecture. Gagandeep Kaur et al (2016) decreases the passive attack on sink node by decreasing the traffic on sink node. The simulation results demonstrate the proposed method can each node will compress their data before sending to cluster head. After compressing, the packet size of node will decrease. This will decrease the traffic overload. Mei Wu et al (2016) proposes a new model to efficiently control cluster-heads using heterogeneous sensors in real-time. It also proposes a generic software framework to feasibly and efficiently meet the needs of the users and applications (e.g., reliability, energy efficiency). The proposed model and its method have been implemented and evaluated in MATLAB, comparing its performance with known protocols for WSNs. The proposed model is suitable for common use because it is superior to other methods by energy efficiency. Sanaa. S et al (2016) introduces an authentication protocol and simple key distributed scheme between sensor nodes. Node mobility has been taken into consideration and the work proposes a re-authentication protocol that is very efficient than the initial protocol. A wireless sensor network (WSN) consists of thousands of sensors and one base station. Sensors are deployed in the network to monitor target area and sense information according to the applied application then send this information to the base station. Roman Lara-Cueva et al (2016) presents a study in order to identify the value range of the main parameters within Carrier Sense Multiple Access (CSMA) defined in IEEE 802.15.4 that guarantee a satisfactory Wireless Sensor Networks (WSN) performance for a possible volcano monitoring application. Moreover, this study performs the comparison between test-bed in outdoor scenarios with the purpose of distinguishing the optimal number of nodes or each gateway according the main constrains given by an existing sensor network for real-time (RT) volcano monitoring system such as sampling time, packet loss, and delay.

\section{RESEARCH MOTIVATION}

Due to the deployment of WSNs in unattended harsh environment conditions, it gets to be unrealistic to charge or replace their batteries. Accordingly, the energy efficient operation of wireless sensors is very important to prolong the lifetime of overall wireless sensor networks. Furthermore, in WSN, there is wireless communication among all the nodes. Moreover, the energy consumed to transmit a message is exceptionally more than the energy needed to receive the same message. Besides all this, the route of each message destined to the base station too decides the network lifetime; for example, any node with depleted battery in short routes to the base station may lead to decreased network lifetime. While, using a long route composed of many sensor nodes can significantly increase the network delay.

- Cluster head election is entirely based on the residual/remaining energy and distance to base station.

- If the $\mathrm{CH}$ will send the data directly to the BS, then there will be much loss of energy for the $\mathrm{CH}$ which is far from the BS. 
- If $\mathrm{CH}$ chooses the multi-hop node (relay node) for data transmission, then the node which is connected to BS will be overloaded with data of all the regions.

- More parameters like number of neighbors, average energy consumption should also be considered before electing the node as cluster head.

\section{CONCLUSION}

The lower cost and easier installation of the WSNs than the wired counterpart pushes industry and academia to pay more attention to this promising technology. Large scale networks of small energy-constrained sensor nodes require techniques and protocols which are scalable, robust, and energy-efficient. Hierarchy provided by clustering techniques is an efficient approach to meet such requirements. Furthermore, data aggregation techniques are only performed by $\mathrm{CHs}$. This research paper focuses on the architecture of wireless sensor networks and their applications. In the future work, the main focus will be kept on implementing the wsn in matlab environment by dividing the network into multiple regions and assigning the cluster head for each region.

\section{REFERENCES}

[1] Y. Xiuwu, Fan Feisheng Zhou Lixing and Z. Feng, "WSN Monitoring Area Partition Clustering Routing Algorithm for Energy-Balanced," IEEE, pp. 80-84, 2016.

[2] S. Bera, S. Misra, Sanku Kumar Roy and Mohammad S. Obaidat, "Soft-WSN: Software-Defined WSN Management System for loT Applications," IEEE, pp. 1-8, 2016.

[3] N. A. M. Alduais, J. Abdullah, J. Abdullah, A. Jamil and L. Audah, "An Efficient Data Collection and Dissemination for IOT based WSN," IEEE, 2016.

[4] O. Singh, V. Rishiwal and M. Yadav, "Energy Trends of Routing Protocols for H-WSN," IEEE, 2016.

[5] R. Kumari and. P. Nand, "Performance Comparison of various Routing Protocols in WSN and WBAN," IEEE, pp. 427-431, 2016.

[6] Hector Kaschel and ohanna Ortega , "Energy efficiency in routing protocols applied to WSN," IEEE, 2016.

[7] Asdianur Hadi and Ida Wahidah, "Delay Estimation using Compressive Sensing on WSN IEEE 802.15.4," IEEE, pp. 192-197, 2016.

[8] Mohd Zaki Shahabuddin, Halabi Hasbullah and Izzatdin A Aziz, "eliminary Framework of Topology Control Algorithm Ahieve Node's Energy Efficiency," IEEE, pp. 259-263, 2016.

[9] Abhaykumar L. Gupta and Narendra Shekokar, "A Novel Approach to Improve Network Lifetime in WSN by Energy Efficient Packet Optimization," IEEE, 2016.

[10] B. Bengherbia, S. Chadli, M. Ould Zmirli and A. Toubal, "A MicroBlaze Based WSN Sink Node Using XBee Transceiver," IEEE, pp. 831-834, 2016.

[11] Gagandeep Kaur, Deepali and Rekha Kalra, "Improvement and Analys Security of WSN From Passive Attack," IEEE, pp. 4520-425, 2016.

[12] M. Wu, H. Liu and Q. Min, "Lifetime Enhancement by Cluster Head Evolutionary Energy Efficient Routing Model for WSN," IEEE, pp. 545-548, 2016.

[13] Roman Lara-Cueva, Rodolfo Gordillo, Liliana Valencia and Diego S. Ben, "Determining the Main CSMA Parameters for Adequate Performance of WSN for Real-time Volcano Monitoring System Applications," IEEE, pp. 1-9, 2016.

[14] Sanaa. S. Abd El dayem and M. R. M. Rizk, "An Efficient Authentication Protocol and Key Establishment in Dynamic WSN," IEEE, pp. 178-182, 2016.

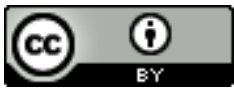

This work is licensed under a Creative Commons Attribution 4.0 International License.

DOI:10.24297/ijct.v16i6.6400 